\title{
Wavelet-Based Response Computation for Base-Isolated Structure under Seismic Excitation
}

\author{
Wensheng Ding1, Zhi Sun ${ }^{2}$ \\ ${ }^{1}$ Department of Bridge Engineering, Tongji University, Shanghai, China \\ ${ }^{2}$ State Key Laboratory for Disaster Reduction in Civil Engineering, Shanghai, China \\ Email: wshding@gmail.com, sunzhi1@tongii.edu.cn
}

Received January 2014

\begin{abstract}
This paper presents a wavelet-based approach for estimating the response of the base-isolated structure under seismic ground motions. The seismic ground motion record is expressed as the multi-scale wavelet coefficients which presents the time-frequency characteristics of the seismic excitation. The wavelet domain governing differential equation between the wavelet coefficients of the excitation and response is derived. Numerical study on a one-storey base isolated structure is performed. The result shows that the wavelet based response computation method is of high precision.
\end{abstract}

\section{Keywords}

Wavelet Transform, Seismic Excitation, Time-Frequency Characteristic, Input-Response Relationship, Wavelet Basis

\section{Introduction}

Earthquakes will usually induce huge disasters to the civil structures. The way to compute structural responses under seismic ground motions has been of continued interest to researchers over the past few decades. Base isolation is a passive device used to reduce earthquake load effect of structures [1]. Compared with other seismic resistant measures, base isolation has good applicability, safety and reliability. Seismic ground motions are generally amplitude and frequency modulated time records [2]. Their evolutions in the time-frequency domain are their main characteristics [3]. The recently developed wavelet analysis has emerged as a powerful tool to analyze variations in time-frequency content [4,5]. It has been widely used for engineering vibration analysis [6].

This paper presents a wavelet-based approach for estimating the response of the equivalent linear base-isolated frame under seismic ground motions. The seismic ground motion record is expressed as the multi-scale wavelet coefficients which presents the time-frequency characteristics of the seismic expression. The wavelet domain governing differential equation between the wavelet coefficients of the excitation and response is derived. Numerical study on a one-storey base isolated structure is performed and presented below. 


\section{Wavelet Transform and Mother Function}

For any function $\psi(t) \in L^{2}(R)$, if it satisfies the admissible condition

$$
C_{\psi}=\int_{R} \frac{|\widehat{\psi}(\omega)|^{2}}{|\omega|} d \omega<\infty
$$

where $\bar{\psi}(\omega)$ is the Fourier transform of $\psi(t)$, the function $\psi(t)$ is called the "mother wavelet". A series of wavelet functions $\psi_{a, b}(t)$, as the dilated and translated version of $\psi(t)$ using scale (or dilation) parameter $a$ and translation parameter $b$, can then be constructed as

$$
\psi_{a, b}(t)=\frac{1}{\sqrt{|a|}} \psi\left(\frac{t-b}{a}\right) \quad a, b \in R ; a \neq 0
$$

The wavelet transform of the function, $f(t) \in L^{2}(R)$, with respect to the basis $\psi(t)$, is defined as

$$
W_{\psi} f(a, b)=<f, \psi_{a, b}>=|a|^{-\frac{1}{2}} \int_{R} f(t) \overline{\left(\frac{t-b}{a}\right)} d t
$$

It can be also expressed in another form as

$$
W_{\psi} f(a, b)=<f, \psi_{a, b}>=\sqrt{a} \int_{R} F(\omega) \overline{\psi_{a, b}(\omega)} d \omega
$$

Here, the $F(\omega)$ is the fourier form of the $f(t)$. It is possible to reconstruct $f(t)$ from its wavelet coefficients $W_{\psi} f(a, b)$

$$
f(t)=\frac{1}{C_{\psi}} \int_{-\infty}^{\infty} \int_{-\infty}^{\infty} \frac{1}{a^{2}} W_{\psi} f(a, b) \psi\left(\frac{t-b}{a}\right) d a d b
$$

This integral has to be discretized for numerical evaluation.

Assuming that

$$
\begin{gathered}
a_{j}=\sigma^{j}, b_{j}=(j-1) \Delta b \\
\Delta a_{j}=\left[\left(a_{j+1}-a_{j}\right)+\left(a_{j}-a_{j-1}\right)\right] / 2, \Delta b_{j}=\left[\left(b_{j+1}-b_{j}\right)+\left(b_{j}-b_{j-1}\right)\right] / 2=\Delta b
\end{gathered}
$$

discretizing the Equation (5) in such a way, the discretized version is thus obtained as

$$
f(t)=\sum_{i} \sum_{j} \frac{K \Delta b}{a_{j}} W_{\psi} f\left(a_{j}, b_{i}\right) \psi_{a_{j}, b_{i}}(t)
$$

where $K$ is a constant with a value equal to $K=\frac{3}{8 \pi C_{\psi}}$.

In this study, the LittleWood-Paley (L-P) mother wavelet function is used as it provides an orthogonal basis with excellent frequency localization characteristics. Its time-domain expression is

$$
\psi(t)=\frac{1}{\pi} \frac{\sin 2 \pi t-\sin \pi t}{t}
$$

the frequency-domain expression is

$$
\hat{\psi}(\omega)=\frac{1}{\sqrt{2 \pi}} \pi \leq|\omega| \leq 2 \pi
$$

As a comparison, the Morlet wavelet mother function and the harmonic wavelet mother function would be chosen. The Morlet wavelet basis is expressed as

$$
\psi(t)=e^{-t^{2} / 2} \cos (5 t)
$$

The frequency-domain expression is

$$
\hat{\psi}(\omega)=\frac{1}{\sqrt{2 \pi}} e^{-\frac{1}{2}(\omega-5)^{2}}
$$


And the Harmonic wavelet basis is expressed as

$$
\psi(t)=\frac{e^{i 4 \pi t}-e^{i 2 \pi t}}{i 2 \pi t}
$$

The frequency-domain expression is

$$
\hat{\psi}(\omega)=\frac{1}{2 \pi} \quad 2 \pi \leq \omega<4 \pi
$$

\section{Wavelet-Based Response Computation of Base-Isolated Structure}

Considering a base-isolated structure subjected to the seismic ground acceleration $z(t)$, if the base-isolation system is set to be equivalent to a linear system, the equation of motion of the structure can be expressed as follow

$$
[m]\{\ddot{x}\}+[c]\{\dot{x}\}+[k]\{x\}=-[m]\{I\} z
$$

where $[m],[c]$ and $[k]$ are the $n \times n$ mass, damping and stiffness matrixes respectively, $\{I\}$ is the $n \times 1$ ground displacement influence vector; $\{x\}$ is the $n \times 1$ displacement vector relative to the ground motion. Assuming the damping is the classically damping [8]. Equation (15) can be transformed into the following $n$ uncoupled equations. The displacements response could then be represented as mode shape vector $\left\{\phi^{(j)}\right\}(j=1,2 \cdots n)$ and generalized modal coordinate $\eta_{j}(t)(j=1,2 \cdots n)$

$$
\{x(t)\}=\sum_{j=1}^{n}\left\{\phi^{(j)}\right\} \eta_{j}(t)
$$

Then, the original equation can be decoupled as a series of equation describing the motion in a particular mode of vibration

$$
\ddot{\eta}_{j}+2 \varsigma_{j} \omega_{j} \dot{\eta}_{j}+\omega_{j}^{2} \eta_{j}=-\alpha_{j} z ; j=1,2 \cdots n
$$

where, $\omega_{j}, \varsigma_{j}, \alpha_{j}\left(=\left\{\phi^{(j)}\right\}^{T}[m][I] /\left\{\phi^{(j)}\right\}^{T}[m]\left\{\phi^{(j)}\right\}\right)$ represent the $j$ th mode natural frequency, damping ratio and participation factor.

Taking the wavelet transform on the both sides of Equation (16), the following relationship is obtained for the response [7]

$$
W_{\psi} x_{i}\left(a_{p}, b_{q}\right)=\sum_{j=1}^{n} \phi_{i}^{(j)} W_{\psi} \eta_{j}\left(a_{p}, b_{q}\right)
$$

where, the $\phi_{i}^{(j)}$ is the ith element of mode shape $\left\{\phi^{(j)}\right\}$. Taking the wavelet transform of both sides of Equation (17) with the chosen wavelet basis $\psi_{a, b}(t)$,

$$
W_{\psi} \ddot{\eta}_{j}(a, b)+2 \varsigma_{j} \omega_{j} W_{\psi} \dot{\eta}_{j}(a, b)+\omega_{j}^{2} W_{\psi} \eta_{j}(a, b)=-\alpha_{j} W_{\psi} z(a, b)
$$

Considering the fast decaying property of the wavelet basis in time domain and it can be shown

$$
W_{\psi} \ddot{\eta}(a, b)=\frac{1}{a^{2}} W_{\ddot{\psi}} \eta(a, b)
$$

The right side of the Equation (20) can be written as

$$
W_{\ddot{\psi}} \eta(a, b)=\frac{\partial^{2}}{\partial b^{2}} \int_{-\infty}^{\infty} \eta(x) \psi_{a, b}(t) d t
$$

Exchanging the integral with the double differentials and applying the Leibnitz rule of differential,

$$
\frac{\partial^{2}}{\partial b^{2}} W_{\psi} \eta(a, b)=\frac{1}{a^{2}} W_{\ddot{\psi}} \eta(a, b)
$$

Substituting the Equation (20) into Equation (22), it can be obtained that

$$
W_{\psi} \ddot{\eta}_{j}(a, b)=\frac{\partial^{2}}{\partial b^{2}} W_{\psi} \eta_{j}(a, b)
$$


Similarly, the following expression can be obtained

$$
W_{\psi} \dot{\eta}_{j}(a, b)=\frac{\partial}{\partial b} W_{\psi} \eta_{j}(a, b)
$$

Substituting the Equation (23) and Equation (24), the Equation (19) is expressed as [9]

$$
\frac{\partial^{2}}{\partial b^{2}} W_{\psi} \eta_{j}(a, b)+2 \varsigma_{j} \omega_{j} \frac{\partial}{\partial b} W_{\psi} \eta_{j}(a, b)+\omega_{j}^{2} W_{\psi} \eta_{j}(a, b)=-\alpha_{j} W_{\psi} z(a, b)
$$

The differential equation shows the relationship between the input wavelet coefficients and the response wavelet coefficients. For a particular $a_{j}$, the Equation (25) can be easily solved, so the wavelet coefficients for the given frequency band can be obtained. It should be noted that the coefficients contain both time and frequency information to understand the time-frequency characteristic of the excitation and response.

\section{Numerical Study}

For a one storey base-isolated structure, Figure 1 shows, the base-isolation system can be equivalent as a linear system. For a base-isolation system of shear displacement ductility ratio $\mu$, strain hardening ratio $\alpha$, and elastic stiffness $K_{u}$, the equivalent stiffness and damping could be expressed as below according to the AASHTO guide specifications [10]

$$
k_{b}=\frac{1+\alpha(\mu-1)}{\mu} K_{u}, \quad \xi_{b}-\xi_{0}=\frac{2(1-\alpha)(\mu-1)}{\pi \mu[1+\alpha(\mu-1)]}
$$

Therefore, the one storey base-isolated structure can be simplified as a 2DOF system with the lumped masses $m_{b}, m_{f}$, the stiffnesses $k_{b}, k_{f}$, and the damping $c_{b}, c_{f}$. Denoting that $T_{b}=2 \pi \sqrt{\left(m_{b}+m_{f}\right) / k_{b}}$, $T_{f}=2 \pi \sqrt{m_{f} / k_{f}}$ which is the vibration period of the non-isolated structure, and $v=m_{f} / m_{b}$. The damping ratio is set to be $\varsigma_{b}=0.10$ and $\varsigma_{f}=0.03$.

The seismic ground motion considered is the seismic ground motion recorded during 1971 San Fernando earthquake. Figure 2 shows the first 15s of ground motion time record and the corresponding wavelet time-frequency characteristic. As presented, the seismic is of an energy concentration in the time duration around $8 \mathrm{~s}$ and in the frequency band of $1-3 \mathrm{~Hz}$.

To compute structural response under earthquake, the wavelet coefficients of structural response in each scale are computed firstly and then transformed into time domain via wavelet reconstruction. For different mother wavelet function, the following parameters are set during the computation: for L-P wavelet mother function, $\sigma=2$, for $\pi \leq a_{j} \omega \leq 2 \pi$, so $j=-5,-4, \ldots 4$; for Harmonic wavelet mother function, $\sigma=2, j=-4,-3, \ldots .5$; for Morlet wavelet mother function, $\sigma=\sqrt{2}, j=-9,-8, \ldots 5$. Figure 3 shows the computed time history of absolute acceleration and relative displacement response of $m_{f}$ by wavelet based computation and time-integration with $v=1, T_{b}=2 s$ and $T_{f}=1 s$. As presented, the computed response histories based on different wavelet basis functions match very well with the computed history by the time-integration.

Varying the natural periods $T_{f}$ from $0.10 \mathrm{~s}$ to the $3.0 \mathrm{~s}$ and fixing $v=1, T_{b}=2 \mathrm{~s}$, the absolute acceleration

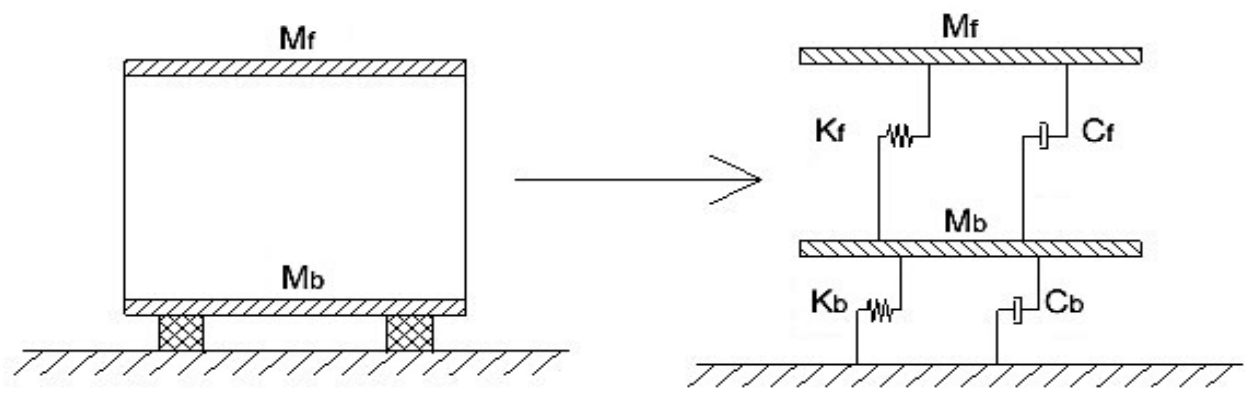

Figure 1. The base-isolated structure. 

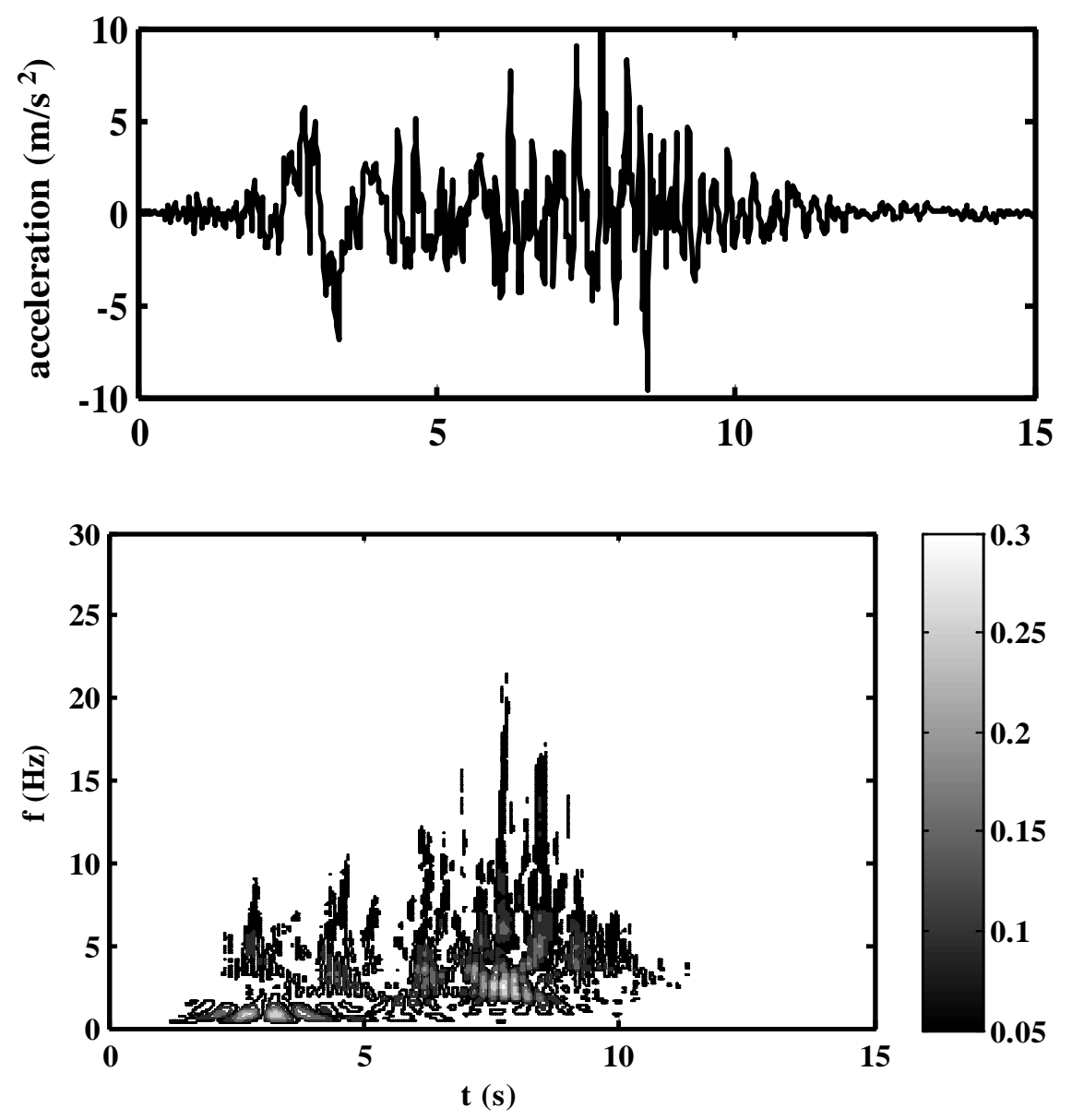

Figure 2. The San Fernando ground motion record and its wavelet representation.

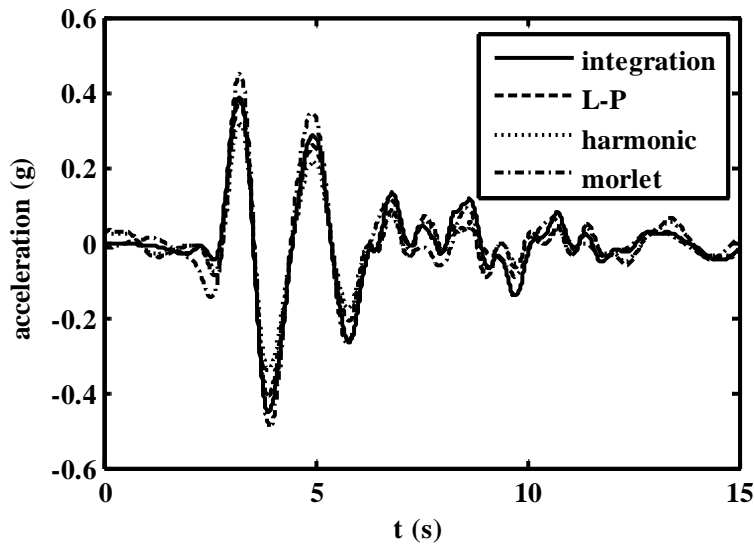

(a)

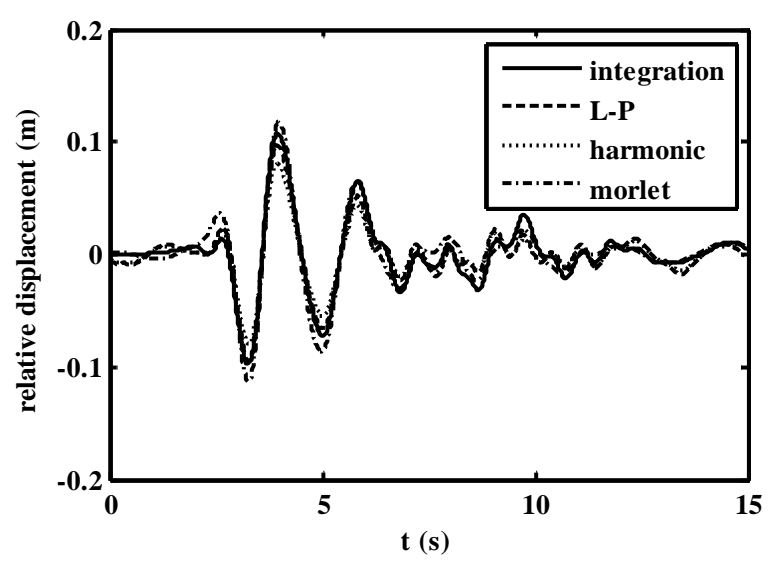

(b)

Figure 3. Comparison of computed acceleration (a) and relative displacement (b) response with $v=1, T_{b}=2 s$ and $\mathrm{T}_{f}=1 \mathrm{~s}$ using the time-integration and wavelet based methods.

and relative displacement response spectrum are computed using different methods and different mother wavelet functions. Figure 4 shows the result. As presented for this study, the response calculated from Morlet wavelet 


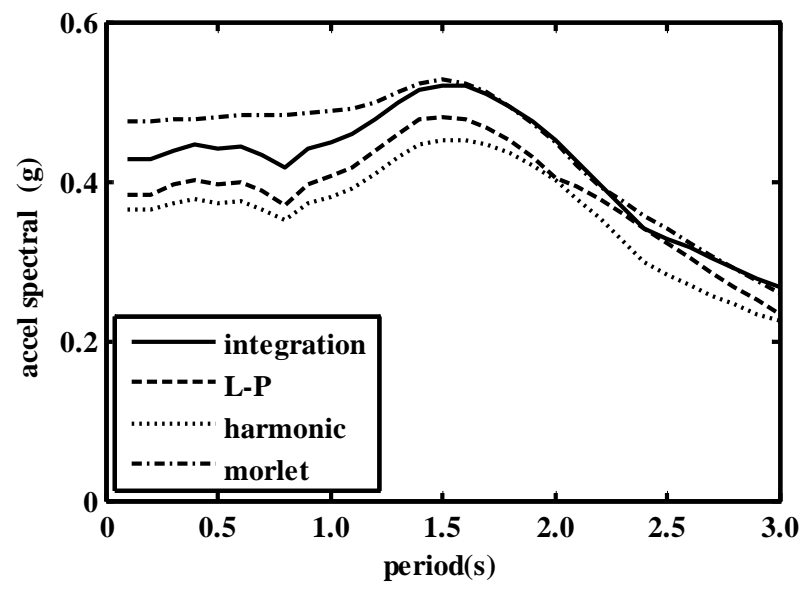

(a)

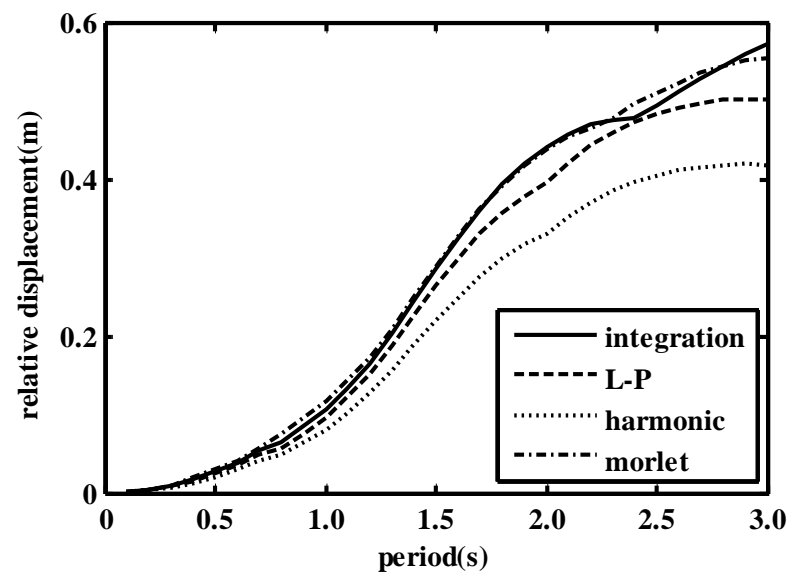

(b)

Figure 4. Comparison of computed acceleration (a) and relative displacement (b) spectra using time-integration and wavelet based methods.

will generally provide an over-estimation and the response calculated from L-P and harmonic wavelet will generally provide an under-estimation; the computation errors for acceleration response spectrum are generally larger than the computation errors for relative displacement response spectrum; the response calculated using L-P wavelet is generally of the best accuracy.

\section{Summary}

This paper presents a wavelet-based approach for estimating the response of the base-isolated structure under seismic ground motions. Numerical study on a one-storey base isolated structure is performed. The results show that the wavelet decomposition can provide orthogonal multi-scale expression of structure governing equation of motion and can be used for structural vibration response computation; the L-P mother wavelet function is of good fast decaying property in time domain and precise localization property in frequency domain and can provide good precision on nonstationary seismic excitation expression as well as on structural seismic response computation.

\section{References}

[1] Takeda, J. (1997) Building Isolation and Vibration Control. China Architecture \& Building Press, Beijing.

[2] Caughey, T.K. and Stumpf, H.J. (1961) Transient Response of a Dynamic System under Random Excitation. Journal 
of Applied Mechanics, 28, 563-566. http://dx.doi.org/10.1115/1.3641783

[3] Basu, B. and Gupta, V.K. (1998) Seismic Response of SDOF System by Wavelet Modeling of Non-Stationary Process. Journal of Engineering Mechanics, 1142-1150. http://dx.doi.org/10.1061/(ASCE)0733-9399(1998)124:10(1142)

[4] Newland, D.E. (1994) Wavelet Analysis of Vibration, Part 1: Theory. Journal of Vibration and Acoustics, ASME, 116, 409-416. http://dx.doi.org/10.1115/1.2930443

[5] Daubechies, I. (1992) Ten Lectures on Wavelets. Society for Industrial \& Applied Mathematics. Philadelphia, Pennsylvania.

[6] Newland, D.E. (1994) Wavelet Analysis of Vibration, Part 2: Wavelet Maps. Journal of Vibration and Acoustics, ASME, 116.

[7] Chopra, A.K. (2007) Dynamics of Structures, Theory and Applications to Earthquake Engineering. Higher Education Press, Beijing.

[8] Basu, B. and Gupta, V.K. (1997) Seismic Response of MDOF System by Wavelet Transform. Earthquake Engineering and Structures Dynamics, 26, 1243-1258. http://dx.doi.org/10.1002/(sici)1096-9845(199712)26:12<1243::aid-eqe708>3.0.co;2-p

[9] Du, P. and Sun, Z. (2010) Wavelet-Based Instantaneous Root-Mean-Square Response Estimation for sdof Systems under Seismic Excitation. Academic Engineering Mechanics, 27, 122-126.

[10] (1991) Guide Specifications for Seismic Isolation Design, American Association of State Highway and Transportation Officials, Washington D.C. 